\title{
Bearing Condition Monitoring based on the Indicator Generated in Time-frequency Domain
}

\author{
Teng Wang ${ }^{1}$, Zheng $\mathrm{Liu}^{2}$ and Guoliang $\mathrm{Lu}^{3}$ \\ ${ }^{1,2}$ Faculty of Applied Science,School of Engineering, The University of British Columbia, \\ Okanagan Campus, Kelowna, VIV 1V7, Canada \\ wangtengsdu@gmail.com \\ zheng.liu@ubc.ca \\ ${ }^{3}$ Key Laboratory of High-efficiency and Clean Mechanical Manufacture of MOE, \\ National Demonstration Center for Experimental Mechanical Engineering Education, \\ School of Mechanical Engineering, Shandong University, Jinan, 250061, China \\ luguoliang@sdu.edu.cn
}

\begin{abstract}
Most condition monitoring systems rely on system-driven generation of indicators or features for early fault detection. However, this strategy requires the prior knowledge on the system kinematics and/or exact structure parameters of monitored system. To address this problem, this paper presents a novel condition monitoring framework where the condition indicator is generated via data-driven method. In this framework, the time-frequency periodogram is extracted from raw vibration signal first. Then, the acquired time-frequency periodogram is mapped by pseudo Perron vector, which is learned from vibration data, to generate the condition indicator. Finally, the bearing can be monitored via analyzing this indicator using gaussian based control chart. Based on experimental results on a publicly-available database, we show the effectiveness of presented framework for early fault detection in the continuous operation of rolling bearing, indicating its great potentials in real engineering applications.
\end{abstract}

\section{INTRODUCTION}

Bearings are vital mechanical parts of most rotating machines, and frequently used in vast domestic and industrial applications. However, they are also fragile components. According to the report, about $41 \%$ of total machine faults are due to bearing (MRWG, 1985). In this context, accurate and reliable bearing condition monitoring plays a critically important role in the predictive maintenance of rotating machinery, which can not only reduce the maintenance cost but ensure

Teng Wang et al. This is an open-access article distributed under the terms of the Creative Commons Attribution 3.0 United States License, which permits unrestricted use, distribution, and reproduction in any medium, provided the original author and source are credited. the reliability of the monitored system during its continuous operation (Hameed, Y. S., Y. M., Ahn, \& C. K., 2009).

Since it has been widely accepted that the degeneration of bearing can be reflected in the bearing vibration signal, most existing schemes aim to monitor the health of bearing via extracting effective features/indicators from vibration signal using signal processing techniques (Javed, Gouriveau, Zerhouni, \& Nectoux, 2014; Dong, Tsui, \& Qiang, 2018). These techniques include time-domain, frequency-domain and time-frequency domain approaches. Among them, timefrequency domain approaches have been an extensively studied area in the literature. The most common timefrequency approaches known in the literature are the shorttime Fourier transform (STFT), the empirical mode decomposition (EMD), the continuous wavelet transform (CWT) and the discrete wavelet transform (DWT). A comprehensive review of such approaches can be found in (Zhipeng, Ming, $\&$ Fulei, 2013). Comparing with time-domain and frequencydomain approaches, time-frequency domain approaches give an insight of operating condition in both time and frequency domian, where frequency spectrum is continuously extracted over time and then analyzed to inspect the dynamic characteristics of bearing vibration signal. The main advantages of time-frequency domain based method can be boiled down as (1) it is promising in analyzing non-stationary signals (Zhipeng et al., 2013), (2) it is able to inspect the incipient fault information with weak amplitude and short duration (Javed et al., 2014).

To extract condition indicators in time-frequency domain, it is crucial to select early fault related frequency components for monitoring. Take the rolling ball bearing for example, bearing failures are mostly due to the defects in the outer raceway 
(OR), inner raceway (IR), or ball. Different type of faults will produce unique frequency component $\left(f_{I R}, f_{O R}, f_{\text {ball }}\right)$ which can be calculated as,

$$
\begin{gathered}
f_{I R}=\frac{n}{2} f_{s}\left(1+\frac{d}{D} \cos \theta\right), \\
f_{O R}=\frac{n}{2} f_{s}\left(1-\frac{d}{D} \cos \theta\right), \\
f_{\text {ball }}=\frac{D}{2 d} f_{s}\left(1-\frac{d^{2}}{D^{2}} \cos ^{2} \theta\right),
\end{gathered}
$$

According to this, a common strategy to evaluate bearing operating status is monitoring these fault-related frequencies, as conducted in many references (Fernández-Francos, MartínezRego, Fontenla-Romero, \& Alonso-Betanzos, 2013; Yaguo, Shantao, Liang, \& Naipeng, 2017; Gerber, Martin, \& Mailhes, 2014; Betta, Liguori, Paolillo, \& Pietrosanto, 2002). If an anomaly appears in one of these frequency sub-bands, it is reasonable to believe the corresponding fault occurs. However, this strategy requires the prior knowledge on exact parameters related to the monitored bearing (such as $\left.\theta, n, f_{s}, D, d\right)$. The acquirement of these parameters is not a trivial work and even impossible in some applications (Combet \& Gelman, 2007).

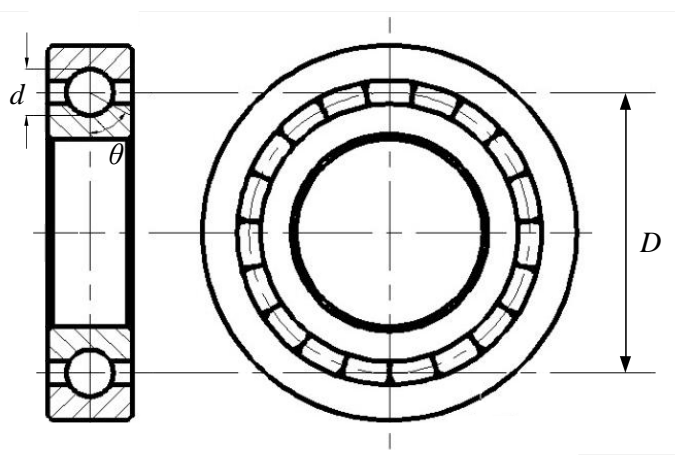

Figure 1. Rolling ball bearing.

To address this problem, this paper presents a novel condition monitoring framework where the condition indicator is generated via data-driven method. Main contributions of this paper are given as follows.

- This paper proposes generating the condition indicator in time-frequency domain by taking into account of the correlations between each pair of frequency components.

- The graph modeling strategy upon time-frequency spectrum is put forward, followed with theoretical interpretation and experimental investigation.

\subsection{Overview of proposed framework}

As shown in fig. 2, given a bearing vibration signal of the whole life cycle, we first extract the time-frequency periodogram and then model it into an undirected weighted graph. By calculating the graph spectrum, the pseudo Perron vector can be acquired as the output of the learning phase. Subsequently, this pseudo Perron vector is used to map the time-frequency periodogram extracted from the vibration signal of monitored bearing, resulting in a one-dimensional condition indicator. Since this indicator is highly related with the operating condition of monitored bearing (the experimental validation is given in Experiment. 3.2 and the theoretical interpretation is given in Appendix. B), the early fault can be timely detected by checking this indicator over time.

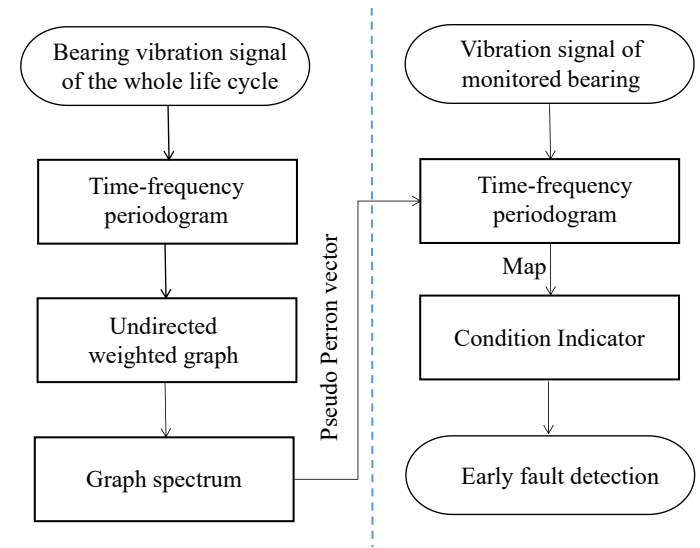

Figure 2. The flowchart of proposed framework.

The rest of this paper is organized as follows. Section. 2 details the proposed framework. In Section. 3, we demonstrate the performance of the proposed framework on a publiclyavailable database, and compare it with a representative method reported in the literature. Finally, Section. 4 concludes this paper and shows our future work.

\section{THE PROPOSED SCHEME FOR BEARING CONDITION MONITORING}

This section describes the proposed framework for bearing condition monitoring which contains four steps: timefrequency periodogram extraction, graph modeling, condition indicator generation, fault decision-making.

\subsection{Time-frequency periodogram extraction}

Derived from the short-time Fourier transform(STFT), timefrequency periodogram estimates the power spectral density of bearing vibration signal. Let us denote the signal as $x(n)$, $n \in[0, N-1]$. The STFT calculation formula in discrete form is given as, 


$$
Y(m, k)=\sum_{n=0}^{N-1} x[n] \omega^{*}[n-m M] e^{-j 2 \pi k \Delta f n}
$$

This transform is discrete in both time and frequency domains where $M$ is time-skip and $\Delta f$ is frequency-skip, $Y(m, k)$ is the output at time $m M$ and frequency $k \Delta f$, the operator $\left(^{*}\right)$ denotes conjugation. The proper window $\omega$ can be selected according to the characteristics of processed signals by considering the amplitude resolution, frequency resolution, time resolution, etc. Note that the Hanning window with a size of $1 * 2000$ is used in this paper.

The short-time periodogram is then calculated by,

$$
P(m, k)=\frac{1}{T}|Y(m, k)|^{2}
$$

Similarly, $P(m, k)$ is the output at time index $0 \leq m \leq M$ and frequency index $1 \leq k \leq K$.

Since it has been well-recognized that the bearing early fault can reflect in the energy distribution over frequency domain, there is a clear motivation to investigate the short-time periodogram of bearing vibration signal, where the power spectral density is continuously extracted over time. However, if the prior knowledge of monitored bearing is not acquirable, how can we extract the fault-related frequency component? This paper proposes mapping the short-time periodogram using pseudo Perron vector which is calculated from graph model.

\subsection{Graph modeling strategy}

A typical graph $G$ consists of a set of nodes and a set of edges, i.e., $G=\{\mathcal{V}, \mathcal{L}\}$. The motivations of employing graph modeling strategy upon time-frequency periodogram are boiled down as follows,

- Graph describes/represents the time-frequency periodogram from a global view, so that both the time information and frequency information are taken into account.

- By introducing edges, the correlation between each pair of frequency component can be described, which is the main innovative point of this paper. Concretely, this paper proposes extracting condition indicator via considering the correlations between each pair of frequency components.

As shown in fig 3, the graph $G$ is structured as follows (Guoliang, Jie, \& Peng, 2018; Teng, Guoliang, \& Peng, 2019).

a) Regard each frequency sample $k=1,2, \ldots, K$ as a node, and connect each pair of two nodes $i$ and $j$ as an edge $\mathcal{L}_{i, j}$ (a) (b)

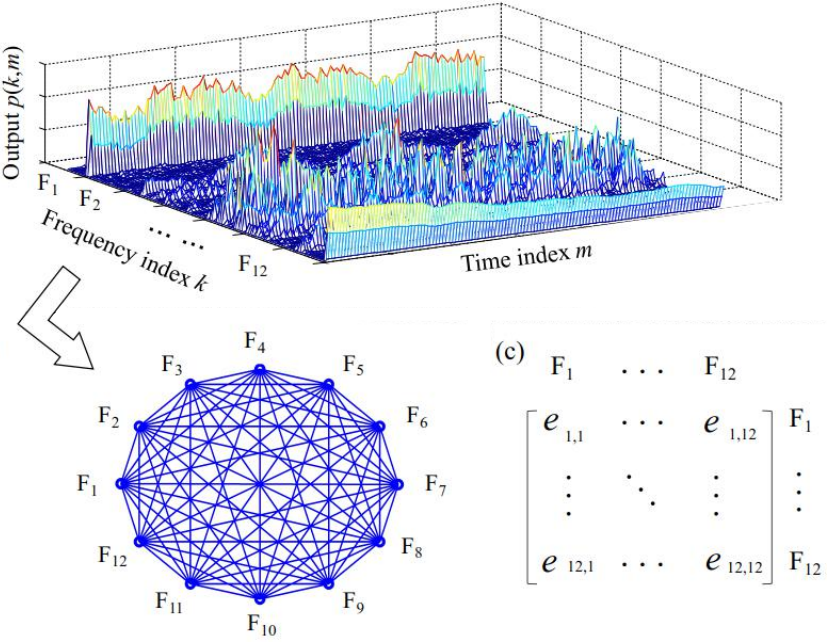

Figure 3. Illustrative example of graph modeling: (a) Timefrequency periodogram that is assumed to contain 12 frequency components. (b) Modeled graph. (c) Adjacent matrix $\alpha$ of the graph.

b) To reflect the correlation between each pair of nodes, calculate the weight $e_{i, j}$ and sign it to edge $\mathcal{L}_{i, j}$;

c) Algebraically express the graph $G$ as an adjacent matrix $\alpha$, i.e., $\alpha=\left\{e_{i, j}\right\}$, which is symmetric and square.

$$
e^{i j}=\operatorname{Cov}\{\aleph(P(i, 1: M)), \aleph(P(j, 1: M))\}
$$

In this paper, the weight of edge is calculated via Eq.(6), where $\operatorname{Cov}(\cdot, \cdot)$ is the covariance. $\aleph$ is mean removal operation. Intuitively, weight $e^{i j}$ describes the covariance between each pair of frequency components.

\subsection{Condition indicator generation}

The condition indicator in this paper is generated by mapping the time-frequency periodogram using pseudo Perron vector. To illustrate this point, let us start with a quick review of graph spectrum.

The employment of matrix for representation of graph has encouraged researchers to study the eigenvalues of these graphs. Graph spectrum is defined by Biggs (Beyer, Goldstein, Ramakrishnan, \& Shaft, 1999) as the eigenvalues of graph in strictly descending order (i.e., $\lambda^{1} \geq \ldots \geq \lambda^{k} \ldots \geq \lambda^{K}$ ) with their corresponding eigenvectors (i.e., $\mathcal{V}^{1}, \ldots, \mathcal{V}^{k} \ldots, \mathcal{V}^{K}$ ).

In the other hand, according to Perron-Frobenius theorem (Berman \& Plemmons, 1994), any nonnegative matrix has a nonnegative principal eigenvalue called the Perron root and any other eigenvalue is strictly smaller than the Perron root in absolute value. The corresponding eigenvector of Perron root is so-called Perron vector which is widely used in many graph based applications such as web page rank, population statis- 
tics. It is worth mentioning that the Perron-frobenius theorem only applies to nonegative matrix. However, as the elements in $\alpha=\left\{e_{i, j}\right\}$ can be negative value in this paper, the $\mathcal{V}^{1}$ can not be regard as Perron vector. In this regard, we name $\mathcal{V}^{1}$ as pseudo Perron vector.

Since pseudo Perron vector $\mathcal{V}^{1}$ is with a size of $1 \times K$ and time-frequency periodogram $P(k, m)$ is with a size of $K \times m$, the condition index $C^{1}(m)$ is acquired by mapping $P(k, m)$ using $\mathcal{V}^{1}$ as,

$$
C^{1}(m)=\mathcal{V}^{1} * P(m, k)
$$

where $m$ is time index.

\subsection{Fault decision-making}

In order to use the condition indicator $C^{1}(m)$ for early fault detection, we first make an assumption upon $C^{1}(m)$ as follows,

Lemma 1: When bearing operates in normal condition, $C^{1}(m)$ is stable. While, the occurrence of fault will cause dramatic fluctuation of $C^{1}(m)$.

Note that the experimental validation and theoretical interpretation of Lemma 1 are given in Experiment. 3.2 and Appendix. B respectively.

Lemma 1 enables the early fault can be detected using gaussian based control chart. Since condition indicator $C^{1}(m)$ is stable when bearing operates normally, we can defined a confidence interval based on a simple assumption that $C^{1}(m)$ follows gaussian distribution (Teng, Guoliang, Jie, \& Peng, 2018). Any early fault will induce $C^{1}(m)$ to exceed this confidence interval. The formulation of fault decision-making is given as follows,

$$
\begin{aligned}
\mathcal{H}_{0}: C^{1}(t) \in A, & \text { i.e., normal operation. } \\
\mathcal{H}_{A}: C^{1}(t) \notin A, & \text { i.e., fault occurs. }
\end{aligned}
$$

where $A=\left[\mu_{t-1}-n \sigma_{t-1}, \mu_{t-1}+n \sigma_{t-1}\right]$ is the confidence interval, $\mu_{t-1}$ is the mean value and $\sigma_{t-1}$ is the standard deviation computed from past data, i.e., $\left\{C^{1}(1), C^{1}(2), \ldots, C^{1}(t-1)\right\} . \quad n$ is the parameter that controls precision and recall. A suitable value of $n$ can be confirmed empirically or by a prior estimation. In the following experiments, $n$ is fixed as 5 empirically.

\section{EXPERIMENT}

In this section, we aim to demonstrate the effectiveness and superiority of the proposed framework. Experiment I and Experiment II are conducted on a publicly-available database to answer the following two questions separately:
- Can the generated indictor reflect the bearing operating condition effectively.

- Does the presented condition monitoring strategy outperforms the representative methods in this area.

\subsection{Experiment materials}

The bearing vibration data used for testing are provided by Center on Intelligent Maintenance Systems (IMS), University of Cincinnati (Hai, Lee, Jing, \& Gang, 2006). As shown in Fig. 4, four bearings were installed on one shaft which was driven by an AC motor and coupled by rub belts. Two accelerometers were mounted on each of bearing to collect the vibration signals in two perpendicular spatial axes. Totally, three times of run-to-failure experiments (Experiment 1 3) were conducted on this platform. It is worth mentioning that the vibration data snapshots were collected with a interval of 10 minutes and we changed the interval into 300 minutes to decrease the computational load. In this study, as exhibited in Appendix. A, all six run-to-failure signals collected from these experiments are tested and their descriptions are given in Table. 1.

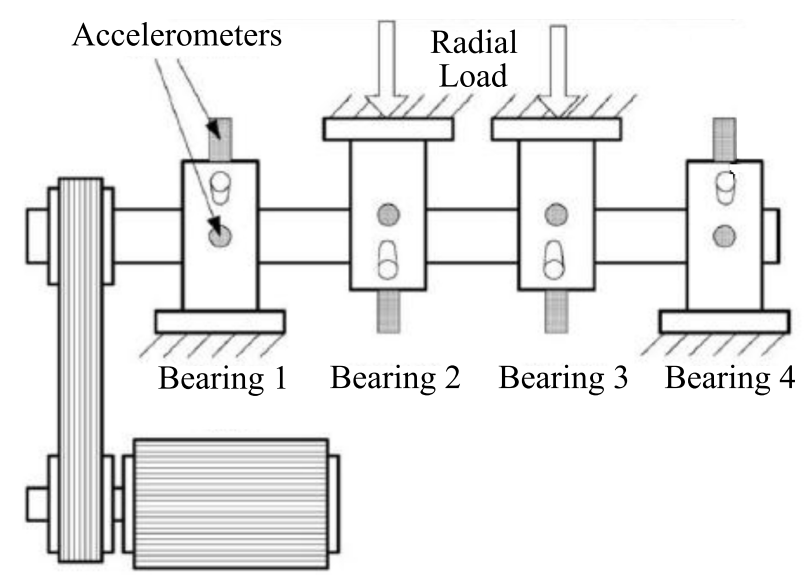

Figure 4. Bearing test platform for fault detection experiment.

\subsection{Experiment I: Performance on early fault detection}

In this Section, the performance of proposed framework is validated. As mentioned in Fig. 2, in the learning phase, the pseudo Perron vector is learned first. Then this vector is used for mapping time-frequency periodogram to acquire the condition indicator in the testing phase. It is worth mentioning that the more similar the learning signal and testing signal are, the better performance will be achieved. Since signal 1 and 2 , signal 3 and 4, signal 5 and 6 are with the same type of fault (seen Table. 1), in this experiment, signals with the same fault type are taken as training signals mutually. For example, signal 1 was used as learning signal to test signal 2 , and vice versa. The condition monitoring results are shown in Fig. 5.

From Fig. 5, we can see that the extracted condition indica- 
Table 1. The description of all six run-to-failure signals in IMS database.

\begin{tabular}{l|l|l|l}
\hline \hline Number & Description & Recording Duration & Fault description \\
\hline \hline Signal 1 & Horizontal vibration signal of bearing 3 collected from Experiment 1. & Approx. 756 h & $\begin{array}{l}\text { At the end, inner } \\
\text { race defect occurred. }\end{array}$ \\
\hline Signal 2 & Vertical vibration signal of bearing 3 collected from Experiment 1. & Approx. 756 h & $\begin{array}{l}\text { At the end, inner } \\
\text { race defect occurred. }\end{array}$ \\
\hline Signal 3 & Horizontal vibration signal of bearing 4 collected from Experiment 1. & Approx. 756 h & $\begin{array}{l}\text { At the end, roller } \\
\text { race defect occurred. }\end{array}$ \\
\hline Signal 4 & Vertical vibration signal of bearing 4 collected from Experiment 1. & Approx. 756 h & $\begin{array}{l}\text { At the end, roller } \\
\text { race defect occurred. }\end{array}$ \\
\hline Signal 5 & Vertical vibration signal of bearing 1 collected from Experiment 2. & Approx. 164 h & $\begin{array}{l}\text { At the end, outer } \\
\text { race defect occurred. }\end{array}$ \\
\hline Signal 6 & Vertical vibration signal of bearing 3 collected from Experiment 3. & Approx. 754 h & $\begin{array}{l}\text { At the end, outer } \\
\text { race defect occurred. }\end{array}$ \\
\hline
\end{tabular}

tor is highly related with the degradation process of tested bearing. The tendency of $C^{1}(m)$ is consistent with the assumption made in Lemma 1, namely when bearing operates normally, $C^{1}(m)$ shows a stable tendency and the early fault can cause a dramatic fluctuation which can be detected by Eq. (8). In summary, all of early faults were successfully detected without any false detections.

\subsection{Experiment II: Comparison with representative works}

As mentioned in Section. 1, the main challenge of condition monitoring from time-frequency domain is extracting faultrelated frequency components. To cope with this issue, existing studies (Gerber et al., 2014; Fernández-Francos et al., 2013; Betta et al., 2002) mainly relies on the selection of frequency sub-bands/components which are highly related with the potential faults. Generally, the selection strategy can be classified into following two categories,

- Prior knowledge based selection: This strategy requires the knowledge on the system kinematics and exact structure parameters of monitored system. For example, using the parameters of bearing structure and rotating speed, the fault-related frequency $f_{I R}, f_{O R}, f_{\text {ball }}$ can be calculated. Then, the sub-bands are determined as $[f-w, f+$ $w], f=f_{I R}, f_{O R}, f_{\text {ball }}, w$ is constant (Yaguo et al., 2017).

- Experience based selection: In this strategy, the decision about the size of the sub-band is made empirically. Generally, two requirements have to be met for sub-band selection (Fernández-Francos et al., 2013): (a) it should not be sensitive to noise and (b) it should be able to accurately concentrate the diagnosis in the band where the fault is significant. Intuitively, if the size of the sub-band is too narrow, the method will be very sensitive to noise. On the other hand, too wide sub-bands would not allow us to accurately localise the exact band where the fault is evident.
Table 2. The detection result of bearing failure

\begin{tabular}{lcc}
\hline \hline \multirow{2}{*}{ Method } & \multicolumn{2}{c}{ Performance indexes } \\
\cline { 2 - 3 } & Precision & Recall \\
\hline 50Hz sub-bands & $0.2 \%$ & $83.3 \%$ \\
100Hz sub-bands & $0.3 \%$ & $100.0 \%$ \\
200Hz sub-bands & $0.4 \%$ & $100.0 \%$ \\
Ours & $100.0 \%$ & $100.0 \%$ \\
\hline \hline
\end{tabular}

Since this paper focus on monitoring the condition without the prior knowledge, we only compare the presented work with the methods in the second category. Concretely, the method reported in (Fernández-Francos et al., 2013) is taken as a competitor, in which the size of sub-band is determined as $200 \mathrm{~Hz}$ and then the average energies of each band are calculated to form a sub-band energy pattern. Finally, the early fault is detected using one-class SVM upon this energy pattern. We implemented this method on the same database used in this paper. Moreover, apart from using the sub-bands with the size of $200 \mathrm{~Hz}$, we also report the detection performance using $50 \mathrm{~Hz}, 100 \mathrm{~Hz}$ sub-bands. Table. 2 summarizes the detection performance of different methods where the detection performance is evaluated with two retrieval indexes defined respectively by,

$$
\begin{aligned}
\text { Precision } & =\frac{\text { Number of Correct Detections of Faults }}{\text { Number of Detections of Faults }} \\
\text { Recall } & =\frac{\text { Number of Correct Detections of Faults }}{\text { Number of True Faults }}
\end{aligned}
$$

The precision indicates the probability that a detection is a true positive; recall is the probability that a true positive can be detected.

It can be seen from Table. 2 that even though sub-band based methods performed well on Recall, these achieved an extremely low performance on Precision i.e., $0.2 \%$ with 
(a)
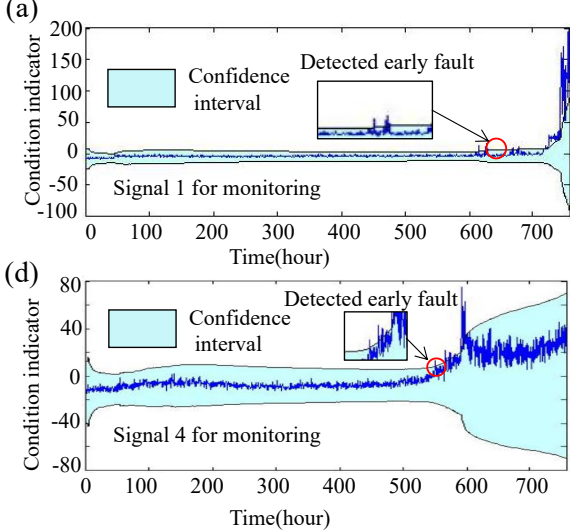

(b)
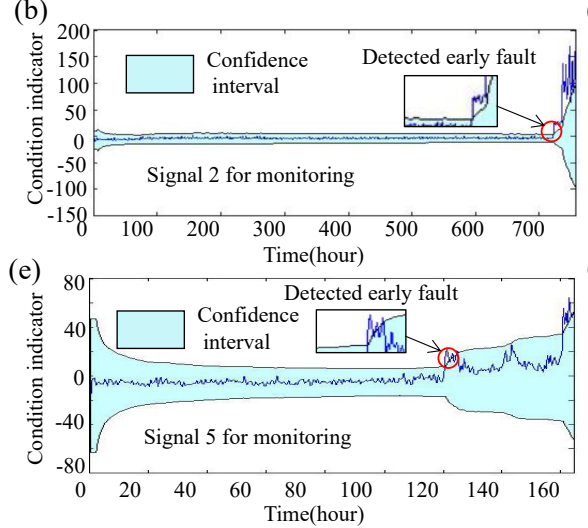

(c)
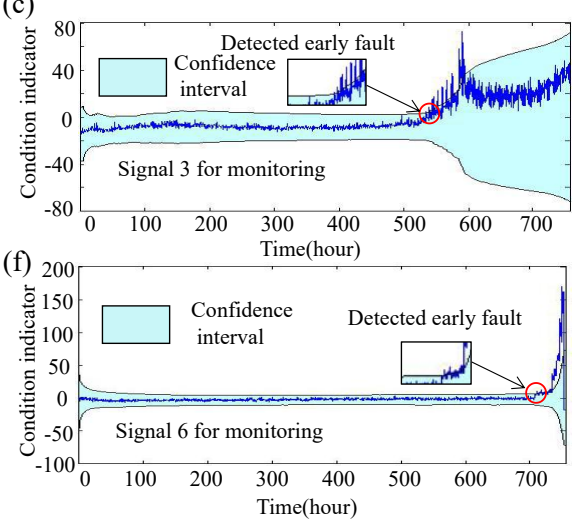

Figure 5. Bearing condition monitoring: (a) Result from signal 1. (b) Result from signal 2. (c) Result from signal 3. (d) Result from signal 4. (e) Result from signal 5. (f) Result from signal 6.

$50 \mathrm{~Hz}$ sub-bands, $0.3 \%$ with $100 \mathrm{~Hz}$ sub-bands, $0.4 \%$ with $200 \mathrm{~Hz}$ sub-bands. The main reason behind this lies in the existence of noises over frequency domain (Carden \& Fanning, 2004). these noises are unrelated with early faults but induce the false detections in the monitoring phase. In comparison, our method achieved a perfect performance in terms of Precision and Recall. It is worth mentioning that our method dramatically outperforms sub-band based method on Precision, which demonstrates that mapping time-frequency periodogram via pseudo Perron vector can effectively distinguish the fault-related frequency information from irregularly fluctuated noises (see Appendix. B for details).

\section{Conclusion}

In this paper, we have presented a novel framework for bearing condition monitoring based on the condition indicator generated in time-frequency domain. In this framework, the time-frequency periodogram is extracted from the raw vibration signal first. Then, the acquired time-frequency periodogram is mapped by pseudo Perron vector to generate the condition indicator. Finally, the bearing can be monitored via gaussian based control chart. In the experiment, we show the effectiveness of proposed framework, which outperforms sub-band selection based method in this area.

Meanwhile, this paper shows the potential of mapping timefrequency spectrum for early fault prognostic. Alone with this line, this work can be expanded to early fault diagnosis, which is the future work of this study.

\section{ACKNOWLEDGMENT}

\section{NOMENCLATURE}

$f_{I R} \quad$ inner raceway fault related frequency component $f_{O R} \quad$ outer raceway fault related frequency component $f_{\text {ball }} \quad$ roller fault related frequency component

$f_{s} \quad$ bearing rotating frequency

$n \quad$ roller number

d roller diameter

$D \quad$ bearing pitch diameter

$\theta \quad$ contact angle

$m \quad$ time index

$k \quad$ frequency index

$P(m, k) \quad$ output of short-time periodogram

$e^{i j} \quad$ edge weight

$\alpha \quad$ adjacent matrix for the representation of a graph

$\mathcal{V}^{1} \quad$ pseudo Perron vector

$C^{1}(m) \quad$ condition index

\section{REFERENCES}

Berman, A., \& Plemmons, R. J. (1994). Nonnegative matrices in the mathematical sciences (Vol. 9). Siam.

Betta, G., Liguori, C., Paolillo, A., \& Pietrosanto, A. (2002). A dsp-based fft-analyzer for the fault diagnosis of rotating machine based on vibration analysis. In Ieee instrumentation and measurement technology conference.

Beyer, K., Goldstein, J., Ramakrishnan, R., \& Shaft, U. (1999). When is nearest neighbor meaningful? In International conference on database theory (pp. 217235).

Carden, E. P., \& Fanning, P. (2004). Vibration based condition monitoring: a review. Structural health monitoring, 3(4), 355-377.

Combet, F., \& Gelman, L. (2007). An automated methodology for performing time synchronous averaging of a gearbox signal without speed sensor. Mechanical sys- 
tems and signal processing, 21(6), 2590-2606.

Dong, W., Tsui, K. L., \& Qiang, M. (2018). Prognostics and health management: A review of vibration based bearing and gear health indicators. IEEE Access, 6, 665-676.

Fernández-Francos, D., Martínez-Rego, D., FontenlaRomero, O., \& Alonso-Betanzos, A. (2013). Automatic bearing fault diagnosis based on one-class $\nu$ svm. Computers and Industrial Engineering, 64(1), 357-365.

Gerber, T., Martin, N., \& Mailhes, C. (2014). Monitoring based on time-frequency tracking of estimated harmonic series and modulation sidebands. In International conference on condition monitoring of machinery in non-stationary operation (pp. 87-98).

Guoliang, L., Jie, L., \& Peng, Y. (2018). Graph-based structural change detection for rotating machinery monitoring. Mechanical Systems and Signal Processing, 99, 73-82.

Hai, Q., Lee, J., Jing, L., \& Gang, Y. (2006). Wavelet filterbased weak signature detection method and its application on rolling element bearing prognostics. Journal of Sound and Vibration, 289(4), 1066-1090.

Hameed, Z., Y. S., H., Y. M., C., Ahn, S. H., \& C. K., S. (2009). Condition monitoring and fault detection of wind turbines and related algorithms: A review. Renewable and Sustainable Energy Reviews, 13(1), 1-39.

Hotelling, H. (1933). Analysis of a complex of statistical variables into principal components. Journal of educational psychology, 24(6), 417-441.

Javed, K., Gouriveau, R., Zerhouni, N., \& Nectoux, P. (2014). Enabling health monitoring approach based on vibration data for accurate prognostics. IEEE Transactions on Industrial Electronics, 62(1), 647-656.

MRWG. (1985). Report of large motor reliability survey of industrial and commercial installations, part $i$ (Vol. 1; Tech. Rep. No. 4). Motor Reliability Working Group.

Teng, W., Guoliang, L., Jie, L., \& Peng, Y. (2018). Graphbased change detection for condition monitoring of rotating machines: Techniques for graph similarity. IEEE Transactions on Reliability, 99, 1-16.

Teng, W., Guoliang, L., \& Peng, Y. (2019). A novel statistical time-frequency analysis for rotating machine condition monitoring. IEEE Transactions on Industrial Electronics. doi: 10.1109/TIE.2019.2896109

Wold, S., Esbensen, K., \& Geladi, P. (1987). Principal component analysis. Chemometrics and Intelligent Laboratory Systems, 2(1), 37-52.

Yaguo, L., Shantao, N., Liang, G., \& Naipeng, L. (2017). A distance metric learning based health indicator for health prognostics of bearings. In 2017 international conference on sensing, diagnostics, prognostics, and control (sdpc) (pp. 47-52).

Zhipeng, F., Ming, L., \& Fulei, C. (2013). Recent advances in time-frequency analysis methods for machinery fault diagnosis: A review with application examples. $\mathrm{Me}$ chanical Systems and Signal Processing, 38(1), 165205.

\section{BIOGRAPHIES}

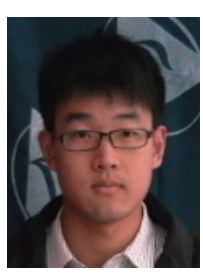

Teng Wang received his bachelor degree from Hefei University of Technology, Hefei, China, in 2016, and received his master degree from Shandong University, Jinan, China, in 2019. He is currently a doctoral candidate at the University of British Columbia (Okanagan campus) at Kelowna, BC, Canada. His research interest includes machine monitoring, prognostics and health management and their applications in industrial system.

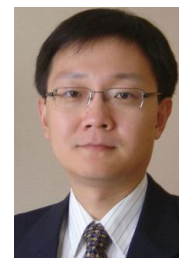

Zheng Liu received his doctorate in engineering from Kyoto University (Kyoto, Japan) in 2000 and earned a second Ph.D. from the University of Ottawa in 2007. From 2000 to 2001, he was a research fellow with Nanyang Technological University (Singapore). He then joined the Institute for Aerospace Research (IAR), National Research Council (NRC) Canada (Ottawa, ON, Canada) as a governmental laboratory visiting fellow (nominated by NSERC). After being with IAR for five years, he transferred to the NRC Institute for Research in Construction, where he held a research officer position. From 2012 to 2015, Dr. Liu worked as a full professor for Toyota Technological Institute at Nagoya, Japan. In August 2015, Dr. Liu joined the University of British Columbia (Okanagan campus) at Kelowna, $\mathrm{BC}$, Canada. His research interests include condition-based maintenance, condition assessment, nondestructive inspection \& evaluation, prognostic health management (PHM), data/information fusion, computer/machine vision, pattern recognition, machine learning, and sensor/sensor network. Dr. Liu is a senior member of IEEE and member of SPIE. He is chairing the IEEE IMS TC-36 and serving as the VP publication of the IEEE Instrumentation and Measurement Society (2016-2017). He holds professional engineer licenses in both Ontario and British Columbia. Dr. Liu also serves on the editorial boards for journal IEEE Transactions on Instrumentation and Measurement, Information Fusion, Machine Vision and Applications, and Intelligent Industrial Systems.

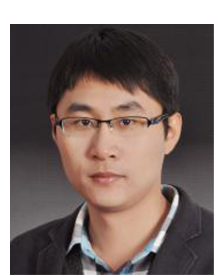

Guoliang Lu received the Bachelor's and Master's degree from Shandong University, Jinan, China, in 2006 and 2009, respectively, and the Ph.D degree from the Graduate School of Information Science and Technology, Hokkaido University, Sapporo, Japan, in March 2013.

He is currently an Associate Professor with Shandong University, Jinan, China. His research interests include signal processing, machine monitoring, machine vision, and visual servo control.

\section{APPENDIX}

\section{Appendix A}

The raw signals used in this paper are exhibited in Fig. 6. 


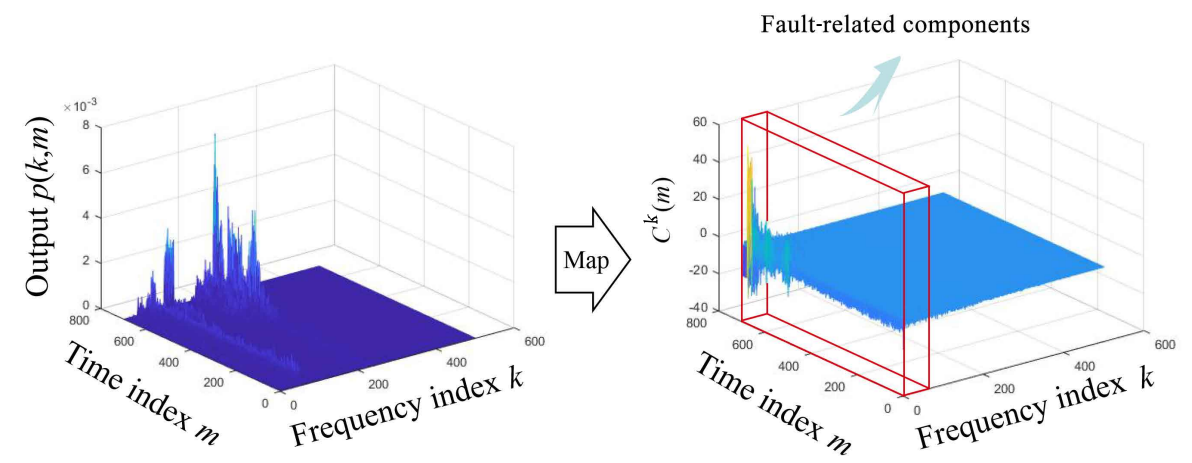

Figure 7. Use $\left\{\mathcal{V}^{1}, \ldots, \mathcal{V}^{k}, \ldots, \mathcal{V}^{K}\right\}$ to map the time-frequency periodogram: (a) Time-frequency periodogram extracted from bearing vibration signal. (b) Fault-related frequency components after mapping, where $C^{1}(m)$ is selected as condition indicator.
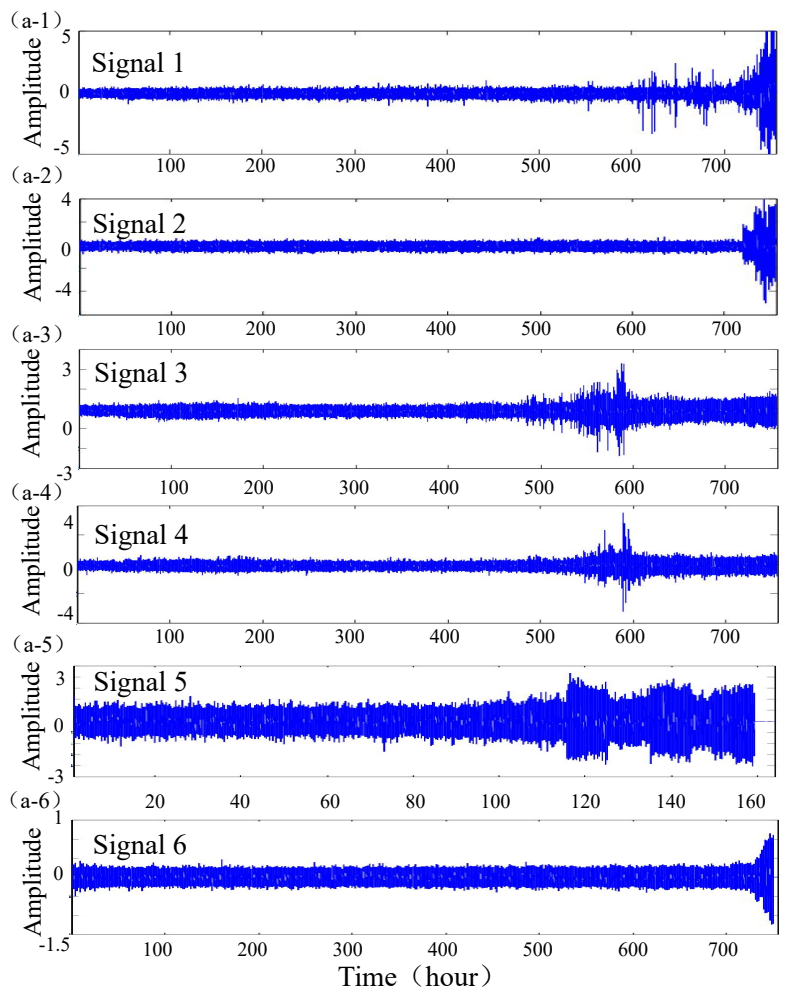

Figure 6. All six run-to-failure signals in IMS database.

\section{Appendix B}

Recall that an assumption is made in Lemma 1 that when bearing operates in normal condition, $C^{1}(m)$ is stable and the occurrence of fault will cause a dramatically fluctuation of $C^{1}(m)$. This appendix presents the theoretical interpretation of Lemma 1.

On the one hand, $\lambda^{1}$ is the eigenvalue and $\mathcal{V}^{1}$ is the eigenvector of graph $\alpha$. The following equation holds,

$$
\alpha \mathcal{V}^{1}=\lambda^{1} \mathcal{V}^{1}
$$

On the other hand, inspired by principal component analy- sis (PCA), let us use a set of orthogonal basis vectors $W=$ $\left\{w^{1}, w^{2}, \ldots, w^{K}\right\}$ to map the time-frequency periodogram $P(m, k)$. The goal is to maximize the variance of frequency in the new space after mapping(Hotelling, 1933; Wold, Esbensen, \& Geladi, 1987), namely,

$$
\begin{gathered}
\max _{W} \operatorname{tr}\left(W^{T} \alpha W\right) \\
\text { s.t. } W^{T} W=I,
\end{gathered}
$$

By using lagrange multiplier method to solve Eq. (10), we can acquire that

$$
\alpha w^{i}=A^{i} w^{i}
$$

Comparing Eq. (9) with Eq. (11), we can draw the conclusion that: (1) the pseudo Perron vector $\mathcal{V}^{1}$ is $w^{1}$. (2)using $\mathcal{V}^{1}$ to map time-frequency periodogram $P(m, k)$ result in the maximum variance of the resulted indicator. More interesting, the maximum variance is $\lambda^{1}$.

This theoretical derivation is not the contribution of this paper as it has be reported in many reference(Hotelling, 1933; Wold et al., 1987). However, this paper believes this maximum variance is induced by early fault of bearing. Moreover, when employing the orthogonal basis vector other than $\mathcal{V}^{1}$ to map the time-frequency periodogram, the lower the variance is, the less information is contained in acquired data. To validate this point, Fig. 7 shows the operation of mapping the timefrequency periodogram using $W=\left\{\mathcal{V}^{1}, \ldots, \mathcal{V}^{k}, \ldots, \mathcal{V}^{K}\right\}$ where signal 5 is taken as an example. It can be seen that without the prior knowledge of bearing, it is almost impossible to select the fault-related frequency sub-band from raw time-frequency spectrum. On the contrast, after mapping, we can aggregate all the frequency information into a specific sub-band as labelled in the red box. The condition indicator used in this paper is $C^{1}(m)$ which has the maximum variance. Meanwhile, note that pseudo Perron vector referred here is not the PCA of time-frequency periodogram. It is the parameter/coefficient can be learned from the time-frequency periodogram of one signal. This parameter can be used to highly the fault-related information in other similar signals. 